\title{
Interactive Visual Tools to Explore Spatio-Temporal Variation
}

\author{
Natalia Andrienko and Gennady Andrienko \\ Fraunhofer AiS -Autonomous Intelligent Systems Institute \\ Schloss Birlinghoven, Sankt-Augustin, D-53754 Germany \\ WWW: http://www.ais.fraunhofer.de/and/
}

gennady.andrienko@ais.fraunhofer.de

\begin{abstract}
CommonGIS is a developing software system for exploratory analysis of spatial data. It includes a multitude of tools applicable to different data types and helping an analyst to find answers to a variety of questions. CommonGIS has been recently extended to support exploration of spatio-temporal data, i.e. temporally variant data referring to spatial locations. The set of new tools includes animated thematic maps, map series, value flow maps, time graphs, and dynamic transformations of the data. We demonstrate the use of the new tools by considering different analytical questions arising in the course of analysis of thematic spatio-temporal data.
\end{abstract}

\section{Categories and Subject Descriptors}

H.5.2 [Information Systems]: User Interfaces - Graphical user interfaces, Interaction styles.

\section{Keywords}

Information visualisation, exploratory data analysis, time-series spatial data, temporal variation, animated maps, time-series analysis.

\section{INTRODUCTION}

Our research work, which is mainly devoted to support of exploratory analysis of spatially referenced data, includes design and implementation of innovative software tools and experimentation with these tools by applying them, individually and in combinations, to various data. The tools are based on traditional techniques and approaches from various disciplines: cartography, statistical graphics, information visualisation, and human-computer interaction. However, we enhance these traditional techniques with high interactivity and manipulability and combine them so as to provide better opportunities for analysis. The results of our work are described in a number of papers, see, for example, [1-3].

In this presentation, we want to demonstrate some new developments not covered by the previous papers. These are tools for exploration of data having both spatial and temporal reference, or, shortly, spatio-temporal data. The work described was partly

Permission to make digital or hard copies of all or part of this work for personal or classroom use is granted without fee provided that copies are not made or distributed for profit or commercial advantage and that copies bear this notice and the full citation on the first page. To copy otherwise, to republish, to post on servers or to redistribute to lists, requires prior specific permission and/or a fee.

AVI '04, May 25-28, 2004, Gallipoli (LE), Italy

(c) 2004 ACM 1-58113-867-9/04/0500..\$5.00 supported by the EU in project GIMMI (IST-2001-34245).

There are different types of spatio-temporal data, in particular, according to the type of changes that occur to them over time [4]:

- existential changes: appearing, disappearing, reviving of objects or/and relationships;

- changes of spatial properties of objects (location, size, shape);

- changes of thematic properties, i.e. values of attributes.

Sometimes only one type of change takes place or is of interest for an analyst, but in many cases one needs to deal with several types simultaneously.

In our presentation, we consider the third type of changes, i.e. changes of thematic properties. We demonstrate the work of our tools using the dataset about crime in USA published by the U.S. Department of Justice. The data are available at the URL http://bjsdata.ojp.usdoj.gov/dataonline/index.cfm. This dataset contains values of 21 numeric attributes referring to 51 states of the USA and to 41 time moments (years from 1960 to 2000). The attributes include the population number and the absolute numbers and rates of crimes of different types, for example, violent crime, property crime, etc.

For a proper design of exploratory tools, we need to anticipate the types of questions that may arise in investigation of the type of data the tools are created for. The major questions concerning thematic changes are:

1 Questions related to a single moment:

1a What were the characteristics of a given location at a given time moment?

1b How were attribute values distributed over a territory at a given time moment?

2 Questions related to two moments:

2a How did the attribute values at the given location change from moment $\mathrm{t}_{1}$ to moment $\mathrm{t}_{2}$ ?

$2 b$ How are changes from moment $t_{1}$ to moment $t_{2}$ distributed over the territory?

2c How did the overall spatial pattern of value distribution change from moment $t_{1}$ to moment $t_{2}$ ?

3 Questions related to a time interval:

3a How do attribute values at a given place vary over time (local behaviour pattern)?

3b Are the behaviours at locations $l_{1}$ and $l_{2}$ similar or different?

3c How are different behaviour patterns distributed over the territory? Are there spatial clusters of similar behaviours?

3d How does the overall spatial pattern of value distribution evolve over time? 
In CommonGIS, we have developed a set of tools that can help an analyst in finding answers to these questions. This includes timecontrolled thematic maps (which, in particular, allow animation), map series, value flow maps, and time graphs. Additionally, we have created tools for dynamic data transformation, which can be used in combination with any of these visual tools to enhance their analytical power.

In the remaining text, we briefly describe the tools and demonstrate their use for data analysis.

\section{EXPLORATION OF SPATIAL PATTERNS AND THEIR CHANGES}

Thematic maps are major tools for investigation of spatial distribution of attribute values. Any technique suitable for cartographic representation of time-irrelevant thematic data can be also used for visualisation of data referring to a single time moment. For example, exploration of distribution of values of a numeric attribute is well supported by choropleth maps with areas painted in different shades according to the respective attribute values.

Time-controlled thematic maps (or, shortly, time maps) are just usual maps (in CommonGIS this means highly interactive and manipulable map displays) representing attribute values at a particular time moment. However, this time moment can be interactively changed, which results in each time map being immediately redrawn. The set of controls shown in Figure 1 is used for changing the display time moment.

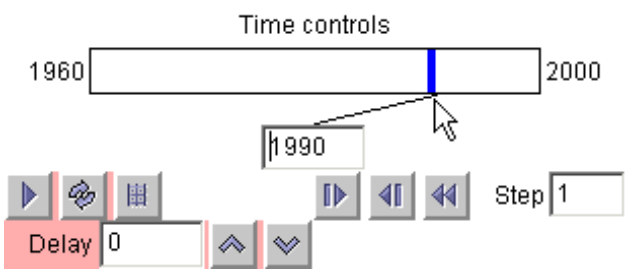

Figure 1. The controls used for choosing the display time moment and for running map animation.

The time moment may be chosen by means of direct manipulation on the time bar (Figure 1, top), pressing the buttons "step forth", "step back", or "return to the start" (Figure 1, lower middle), or by entering the desired time moment in the text field (Figure 1, centre). Besides, it is possible to run automatic map animation and regulate its speed by setting the delay between map updates.

Time maps support first of all finding answers to questions related to a single time moment, which can be interactively selected. A map as a whole shows the spatial distribution of attribute values at this time moment (question 1b). In order to know the attribute values referring to a particular location (question 1a), it is sufficient to point on this location with the mouse cursor, and the values will be shown in a popup window. Map animation allows the user to observe the evolution of the spatial pattern of value distribution (question 3d). However, while map animation is useful for revealing general trends in the development of spatial patterns, time maps are not sufficiently productive for a more detailed investigation of changes that occurred from moment $t_{1}$ to moment $t_{2}$ (question 2c). For comprehensive comparison of states at two or more time moments these states need to be displayed on the screen simultaneously.
Map series represent the distribution of values of the same attribute(s) at several selected time moments thus enabling convenient comparisons. The maps are displayed in a common panel and are manipulated all together (Figure 2). However, the number of maps that can be simultaneously displayed and investigated is limited by the screen size as well as by human's perceptual capabilities. Hence, only a few representative time moments are usually selected for a detailed comparative study. An investigation of pattern changes using a time map can help in detecting the moments that require an additional attention.

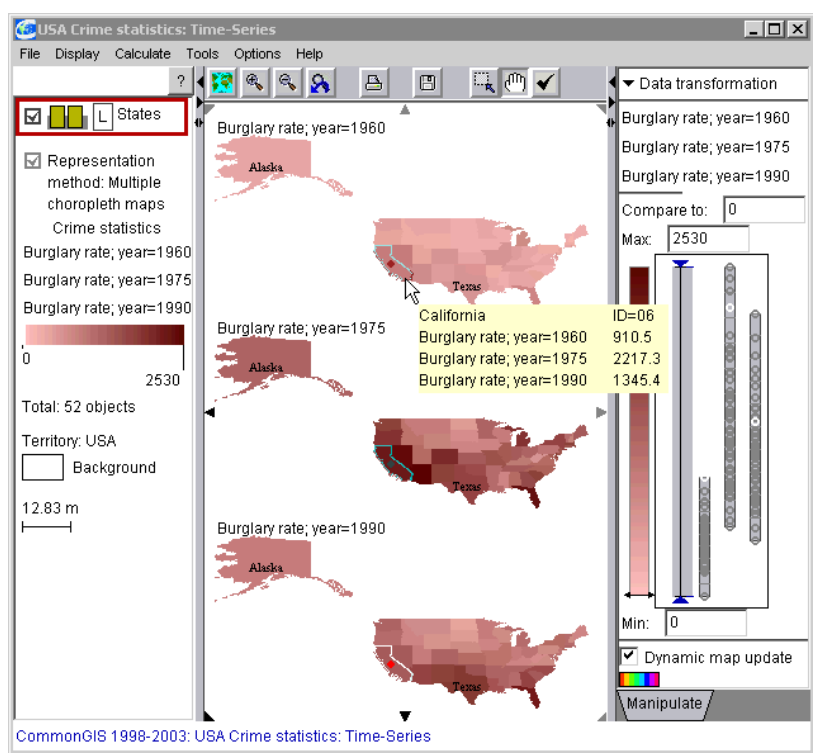

Figure 2. Multiple maps displayed in a common panel and manipulated through a common set of controls support comparison of value distributions at several time moments.

Besides questions concerning the change of the overall spatial pattern (2c), map series displays, due to their interactivity, allow the user to evaluate local changes (question 2a). When the mouse cursor is positioned over an area on one of the maps, the attribute values for all time moments represented in the whole panel are shown in a popup window (see Figure 2). Still more advanced opportunities for investigating local changes and their distribution over the territory are provided by the tools for dynamic data transformation.

\section{EXPLORATION OF LOCAL CHANGES AND THEIR SPATIAL DISTRIBUTION}

Tools for dynamic data transformation can be applied to numeric attributes. These tools (shortly called transformers) are automatically attached to any map display representing numeric attributes and to some non-cartographical displays. Transformers give an opportunity to replace the representation of the original attribute values by that of transformed values without the necessity to build another display. For example, having a map representing burglary rates in year 1990, an analyst may wish to compare these rates to those in year 1989. After the selection of the corresponding transformation from the list of possible transformations, the map is redrawn to represent the differences between the values in 1990 and the corresponding values in the previous year. If this is an unclassified choropleth map, different colours are used for the positive and negative differences (Figure 
3). Hence, the analyst can immediately distinguish the areas where the values increased from those where the values decreased. If this is a map with bar diagrams, the positive differences are represented by upward-oriented bars, and the negative by downward oriented bars. The analyst can easily switch the transformation off and return to the original values.

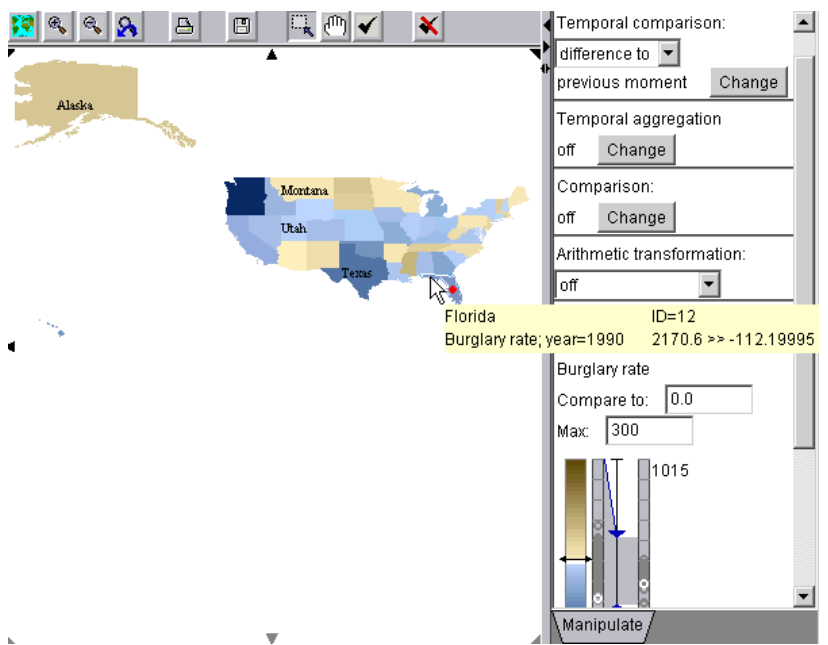

Figure 3. The transformation "temporal comparison" is applied to burglary rates. The original attribute values at a selected time moment (year 1990) have been replaced by the differences to the corresponding values for the previous year.

The following attribute transformers are available in CommonGIS:

- Temporal comparison. The transformer computes the amounts of change, i.e. the differences or ratios between the values for each moment and the values for the previous moment or any user-selected moment. An example of the use of this transformer is shown in Figure 3.

- Temporal aggregation mitigates random fluctuations in data and facilitates revealing long-term trends and cycles. The user may control the length of the interval used for smoothing the data. It is also possible to switch between seeing the smoothed values and the differences between them and the original values (residuals).

- Comparison (time-irrelevant). The transformer facilitates the comparison of the original values with the overall mean, median, or any selected value as well as with the value associated with a selected object. For this purpose, it either subtracts the reference value from the original values or divides the original values by the reference value. In a timecontrolled map, this transformation is applied to each time moment taking into account the changes of the reference value.

- Arithmetic transformation may be useful, for example, when an attribute has only a few high values standing far apart from the remaining, relatively low values. Replacing the original values by their logarithms makes the representation more expressive. Some other arithmetic transformations are also supported.

It is possible to use several transformers in a row. In this case, every transformer starting from the second one applies its transformation to the results produced by the previous transformer. It is important that the user can switch any transformation on or off or change its parameters, and the results are immediately represented on the current map or graph, without requiring the user to rebuild it.

The operation of temporal comparison is especially supportive for the investigation of local changes from moment $t_{1}$ to moment $t_{2}$ (question 2a). Moreover, after this transformation is applied to a map, the map exposes the distribution of changes over the whole territory and thus helps to answer the question $2 \mathrm{~b}$. When the temporal comparison is applied to a time-controlled map, the user can easily switch between different time moments and see the changes at this time moments in comparison to the previous moment or to a fixed selected moment. The transformation also takes place in the course of map animation.

\section{EXPLORATION OF LOCAL BEHAVIOURS}

Neither time-controlled maps nor map series are suitable for the exploration of temporal variation of attribute values (we call it behaviour) at any particular location. Therefore, we have designed and developed additional exploratory tools that can help an analyst to find answers to questions 3a, 3b, and 3c.

Value flow maps (Figure 4) represent the evolution of attribute values (behaviour) at each location by means of special diagrams called value flow symbols. In such a diagram, the horizontal dimension corresponds to the time interval under investigation, and the vertical dimension is used for representing values of a numeric attribute at each moment from the interval. Hence, the variation of the symbol height from left to right represents the variation of the attribute values over the time interval. The map manipulation controls (not shown in Figure 4, for space reasons), allow the user to flexibly select any subintervals of the whole interval the dataset refers to. Besides local behaviours (question 3a), value flow maps show the distribution of different behavioural patterns over the territory (question 3c).

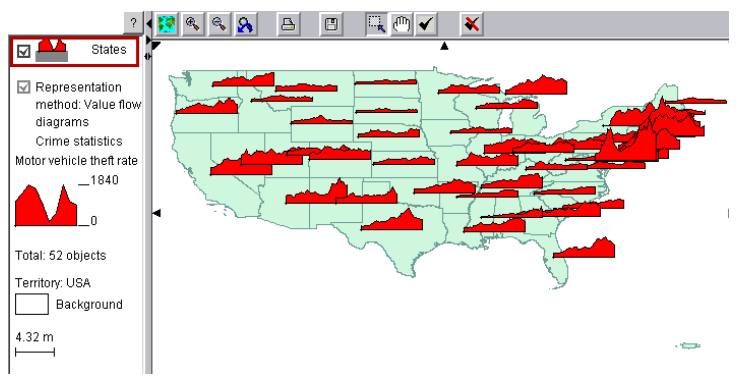

Figure 4. The value flow symbols represent the evolution of attribute values (behaviour) at each location.

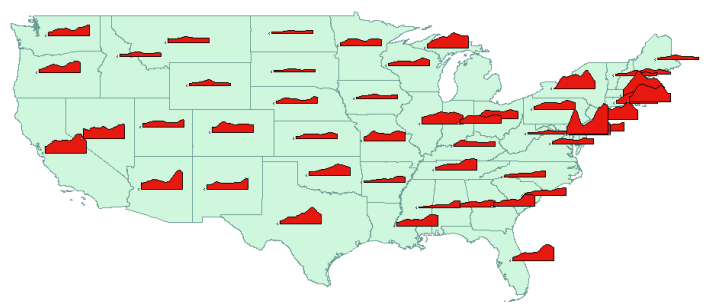

Figure 5. Temporal smoothing on a value flow map allows one to disregard small value fluctuations and see development trends. 
The above-described temporal aggregation transformation can be aptly applied to a value flow map (Figure 5).

Another useful transformation would be comparison to the country's mean or median at each time moment. As can be seen from Figure 6, this transformation can make the patterns of behaviour distribution more prominent.

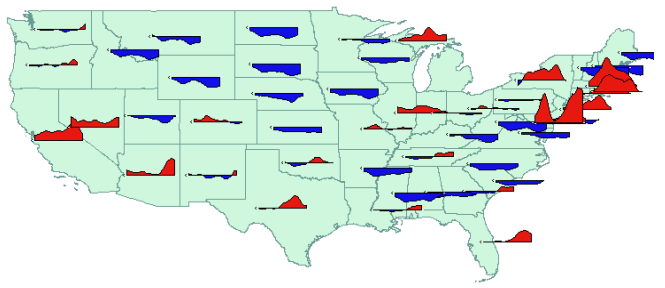

Figure 6. Comparison to the country's mean: the attribute values for each moment are replaced by their differences to the country's mean at the same moment. Red colour corresponds to positive differences and blue to negative.

Although one can compare local behaviours at different locations (question 3b) using a value flow map, it is only suitable for a rough comparison of symbol shapes and sizes. If more precise evaluation of differences is required, this can be better done with a time graph display (Figure 7).

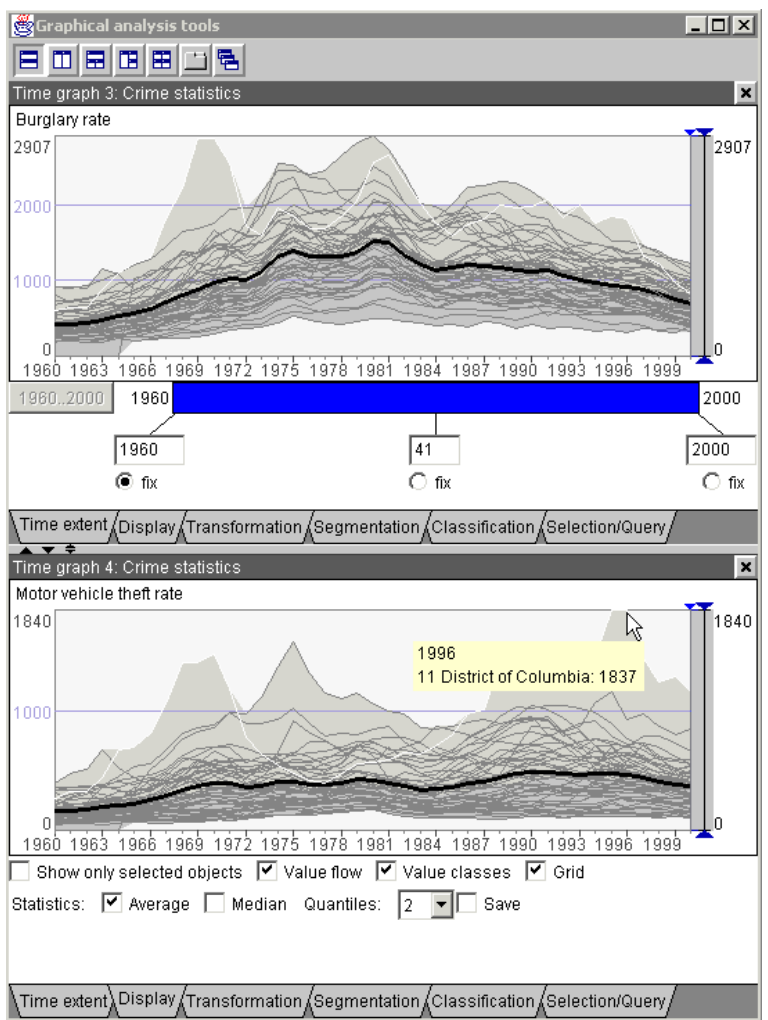

Figure 7. A time graph puts all local behaviours together for enabling more convenient comparisons.

On a time graph, all local behaviours are represented in a common coordinate system. This makes it easier to estimate the amount of difference between two behaviours at any time moment. On the other hand, line cluttering makes it difficult to focus on particular behaviours. To simplify this, the time graph display provides a set of controls for flexible selection of the information to be present on the graph (Figure 7, bottom). Thus, it is possible that only behaviours for user-selected locations are shown, which makes their comparison quite easy.

Besides lines for individual objects (locations), a time graph can also represent the "average behaviour" (the line obtained by connecting each year's means) or the "median behaviour" (obtained from each year's medians). This helps an analyst to understand the general development trends for a territory as a whole. Time graphs are also suitable for comparing variations of two or more attributes.

A time graph display provides a range of advanced exploratory facilities, which will be demonstrated at the conference.

\section{CONCLUSION}

In our work on design and development of software tools for exploratory data analysis, we follow the systematic approach that can be called task-analytical. First, we take a particular data set and consider it as a representative of a certain class of data. From this perspective, we try to reveal the general structure and properties of this class of data. On this basis, we enumerate the types of tasks, or questions, that can arise in exploration of such data. Then we try to find a combination of techniques to support this set of tasks as fully as possible. As a basis, we mostly use traditional techniques from cartography and statistical graphics but enhance their analytical power with interactivity and transformability. Technique combination often produces synergistic effects.

In this presentation, we have demonstrated the set of tools we created for analysis of time-variant thematic data referring to spatial locations. We listed the major exploratory tasks relevant for this class of data and explained which tasks each of the tools is intended to support. While we believe that the tools are effective for the tasks they are designed for, this needs to be tested by users performing data analyses in various problem domains.

\section{REFERENCES}

[1] G. Andrienko and N. Andrienko, "Interactive Maps for Visual Data Exploration", International Journal of Geographical Information Science, 13 (4), 1999, pp.355374.

[2] N. Andrienko and G. Andrienko, "Interactive Visual Tools for Spatial Multicriteria Decision Making”, in M. De Marsico, S. Levialdi, E. Panizzi (Eds.) Proceedings of the Working Conference on Advanced Visual Interfaces AVI 2002, Trento, Italy, May 22-24, 2002, ACM press, 2002, pp.129-132

[3] G. Andrienko and N. Andrienko, "Informed Spatial Decisions through Coordinated Views", Information Visualization, 2 (4), 2003, pp.270-285

[4] C. Blok, "Monitoring Change: Characteristics of Dynamic Geo-spatial Phenomena for Visual Exploration", in Ch.Freksa et al. (eds) Spatial Cognition II, LNAI 1849, Berlin Heidelberg, Springer-Verlag pp.16-30 